\title{
Registro de Xenopsylla cheopis como hospedero intermediario natural de Hymenolepis diminuta en Lima, Perú
}

\author{
Xenopsylla cheopis record as natural intermediate host of Hymenolepis diminuta \\ in Lima, Peru
}

\author{
Inés Gárate, Paolo Jiménez, Karen Flores y Bertha Espinoza
} Laboratorio de Parasitología Huma-
na y Animal, Facultad de Ciencias
Biológicas, Universidad Nacional
Mayor de San Marcos. Apartado
110058, Lima 11, Perú.
Email Inés Gárate: igarateca@
yahoo.com

\begin{abstract}
Resumen
Con el objetivo de determinar el rol de las pulgas de las ratas en el ciclo biológico de Hymenolepis diminuta, entre noviembre del 2009 y marzo del 2010, se capturaron 27 ratas del Cercado de Lima y examinaron sus ectoparásitos, empleando los procedimientos parasitológicos estándares. De las ratas examinadas, 22 (81,5\%) de las ratas examinadas estuvieron infectadas con el parásito $H$. diminuta, el $85,2 \%$ de ratas estuvo infestado por sifonápteros de la especie Xenopsylla cheopis. De las 158 pulgas encontradas, el 12\% albergaba cisticercoides en su hemocele, los que al ser inoculados en ratas albinas de la cepa Holtzman, desarrollaron hasta el estadio de adultos de la especie Hymenolepis diminuta. Los resultados del presente trabajo son concluyentes y constituyen el primer registro del rol de $X$. cheopis como hospedero intermediario natural en Perú, por lo que es necesario reconsiderar los factores de riesgo y las medidas de prevención.
\end{abstract}

Palabras claves: Parasitosis, hymenolepiosis, sifonápteros, cisticercoide.

\section{Abstract}

In order to determine the role of rat fleas in the life cycle of Hymenolepis diminuta, between November 2009 and March 2010, 27 rats were captured in Cercado de Lima and their ectoparasites were examinated, using standard parasitological procedures. In examined rats, $22(81.5 \%)$ were infected with the parasite $H$. diminuta, $85,2 \%$ were infested with siphonapters of the species Xenopsylla cheopis. Of the 158 fleas that were found, $12 \%$ housed cysticercoids in its hemocoel, which were inoculated into albino rats of Holtzman strain, and then developed to adult stage of Hymenolepis diminuta. The results of this study are conclusive and constitute the first record of the role of $X$. cheopis as natural intermediate host in Peru, making it necessary to reconsider the risk factors and preventive measures.

Keywords: Parasitosis, hymenolepiosis, siphonaptera, cysticercoid.

\section{Introducción}

El céstodo Hymenolepis diminuta (Rudolphi 1819) Weinland, 1858 es un parásito cosmopolita que en el estadio adulto se encuentra en ratas y accidentalmente en el hombre. Su ciclo biológico se desarrolla con la intervención de un artrópodo, el cual se infecta al ingerir los huevos de $H$. diminuta que se encuentran en las heces de las ratas parasitadas (Smyth 1963, Roberts et al. 2009).

Experimentalmente, se ha demostrado que 90 especies de artrópodos pueden servir como hospederos intermediarios (Cáceres y Guillén 1972). Aunque Joyeux (1916) seńaló a Tenebrio molitor, a Ceratophyllus fasciatus y Xenopsylla cheopis como las fuentes usuales de infestación de las ratas, los coleópteros del género Tribolium son los señalados por la literatura científica como los más frecuentes en las infecciones de ratas y del hombre. Tribolium alberga en su hemocele al estadio cisticercoide, que es la forma infectante para el hospedero definitivo vertebrado (Roberts et al. 2009). En Perú, Tribolium castaneum y T. confusum son considerados importantes hospederos intermediarios de $H$. diminuta, según señalan Arrojo et al. (2004), quienes lograron infectar coleópteros de la especie T. castaneum con huevos de $H$. diminuta, observando su alta susceptibilidad a la infección. Sin embargo, hasta el presente, no se han encontrado artrópodos de este género naturalmente infectados en este país.

En Perú se han examinado 3873 ejemplares vivos pertenecientes a 15 especies de insectos con el objeto de descubrir a los hospederos intermediarios naturales (Cáceres \& Guillén 1972); así también se han realizado ensayos experimentales, empleando varios artrópodos, con la finalidad de detectar hospederos intermediarios potenciales. De manera similar a lo realizado por Yamada et al. (1936) en Japón, Cáceres y Guillén (1972) utilizando huevos y proglótidos grávidos de H. diminuta lograron infectar individuos de $X$. cheopis, pero no encontraron esta relación naturalmente.

En Perú existen pocos casos de infección humana por $H$. diminuta, sin embargo la prevalencia es alta en algunos lugares: 7,8\% en escolares entre 6 y 15 años de Magdalena en Cajamarca (Náquira et al. 1973) y 7,1\% en la población de Tingo María en Huánuco (Huiza et al. 1986), deduciendo que los alimentos, almacenados e infestados con ratas y coleópteros, al consumirse sin la adecuada cocción, constituyen el medio de infección.

Con el objeto de determinar el rol que juega Xenopsylla cheopis en el ciclo biológico de Hymenolepis diminuta, entre noviembre del 2009 y marzo del 2010, se examinaron 27 ratas y sus ectoparásitos procedentes del Cercado de Lima-Perú.

\section{Material y métodos}

Entre noviembre del 2009 y marzo del 2010, se investigaron 27 ratas peridomésticas y sus ectoparásitos del Cercado de Lima-Perú.

Los roedores fueron capturados empleando una trampa tipo Sherman, trasladados al Laboratorio de Parasitología Humana y Animal de la Facultad de Ciencias Biológicas de la Universidad Nacional Mayor de San Marcos y eutanizados con cloroformo en cámaras cerradas. Los animales fueron examinados en una bandeja de fondo blanco para colectar sus ectoparásitos con ayuda de un peine y un pincel (Pozo et al. 2005), registrándose su sexo, peso (empleando una balanza marca Soehnle ultra 2.0, con un mínimo de $0,1 \mathrm{~g}$ de precisión), y longitud del cuerpo sin considerar el tamańo de su cola (empleando una regla con un 
Tabla 1. Parasitismo por Hymenolepis diminuta y Xenopsylla cheopis en Rattus norvegicus, Lima-Perú.

\begin{tabular}{|c|c|c|c|c|c|c|c|}
\hline \multirow{2}{*}{ Parásitos } & \multirow{2}{*}{$\begin{array}{c}\text { Prevalencia } \\
\text { total }(\%) \\
n=27\end{array}$} & \multicolumn{2}{|c|}{ Prevalencia según sexo (\%) } & \multirow{2}{*}{$\begin{array}{l}\text { Frecuencia } \\
\text { total }\end{array}$} & \multicolumn{2}{|c|}{ Intensidad } & \multirow{2}{*}{ Localización } \\
\hline & & $\begin{array}{c}\text { Hembras } \\
n=12\end{array}$ & $\begin{array}{c}\text { Machos } \\
n=15\end{array}$ & & Mayor & Menor & \\
\hline \multicolumn{8}{|l|}{ Céstodo } \\
\hline Hymenolepis diminuta & 81,5 & 83,3 & 80 & 22 de 27 & 24 & 1 & $\begin{array}{c}\text { Intestino delgado (6 } \\
\text { individuos de una rata } \\
\text { en intestino grueso) }\end{array}$ \\
\hline \multicolumn{8}{|l|}{ Ectoparásito } \\
\hline Xenopsylla cheopis & 85,2 & 83,3 & 86,6 & 23 de 27 & 57 & 1 & Pelaje \\
\hline
\end{tabular}

mínimo de $1 \mathrm{~mm}$ de medición). Las ratas fueron identificadas en el Museo de Historia Natural de la Universidad Nacional Mayor de San Marcos.

Las ratas se dividieron según las categorías pesos estándar (Grupo I, con menos de 250 g; Grupo II, con 250 g a más) y longitud (Grupo I, con menos de $20 \mathrm{~cm}$; Grupo II, con 20 cm a más); luego se les disecó y se extrajo cuidadosamente los intestinos para ser colocados en una placa de Petri con solución salina. Se examinó el contenido de las últimas porciones del intestino grueso empleando el método directo simple con el fin de detectar los huevos de $H$. diminuta.

Con ayuda de un microscopio estereoscópico, se hizo un corte a lo largo de los intestinos y se colectaron los parásitos. Éstos fueron trasladados a una placa de Petri conteniendo solución salina a $0,85 \%$, para su identificación y registro de sus características morfométricas.

Los ectoparásitos de cada rata fueron contados, depositados sobre una luna de reloj con una gota de solución salina, observados con el microscopio estereoscópico, e identificados según Traub (1952) y DIGESA (2002).

Las pulgas fueron examinadas cuidadosamente por exposición de su hemocele. Los cisticercoides hallados fueron contados, medidos (empleando un ocular micrométrico) y fotografiados con una cámara fotográfica (marca Nikon Coolpix.P5100, de $12 \mathrm{MP}$ ) adaptada a un microscopio de luz Carl Zeiss; los especímenes representativos fueron depositados en la helmintoteca del Laboratorio de Parasitología Humana y Animal de la Facultad de Ciencias Biológicas de la UNMSM.

Para comprobar que los cisticercoides extraídos del hemocele de las pulgas correspondían a la forma larvaria de Hymenolepis diminuta, éstos se inocularon por vía oral, empleando una cánula, a Rattus norvegicus, cepa Holtzman, procedentes del bioterio

Tabla 2. Intensidad de infección parasitaria por Hymenolepis diminuta en Rattus norvegicus.

\begin{tabular}{ccc}
\hline $\begin{array}{c}\text { Roedores } \\
\text { parasitados } \mathrm{n}=22\end{array}$ & $\begin{array}{c}\text { Parásitos por } \\
\text { roedor }\end{array}$ & $\%(\mathrm{n}=22)$ \\
\hline 8 & 1 & 36,4 \\
3 & 6 & 13,6 \\
3 & 8 & 13,6 \\
2 & 3 & 9,1 \\
2 & 10 & 9,1 \\
1 & 2 & 4,5 \\
1 & 4 & 4,5 \\
1 & 9 & 4,5 \\
1 & 24 & 4,5 \\
\hline
\end{tabular}

del Instituto Nacional de Salud (INS), con certificado sanitario, a los que a partir del décimo día se les realizó exámenes de heces.

Finalmente, para medir el grado de asociación entre el peso y longitud de la rata con el hecho de que esté parasitada con $H$. diminuta, se realizó un análisis de dispersión bivariado empleando un gráfico de dispersión.

\section{Resultados}

Los 27 roedores, capturados en el Cercado de Lima y examinados en este estudio, pertenecen a la especie Rattus norvegicus (Berkenhout), 12 hembras (44,4\%) y 15 machos (55,6\%); $22(81,5 \%)$ estuvieron infectados con el parásito $H$. diminuta (83,3\% de hembras y $80 \%$ de machos).

En los grupos de animales con mayor peso (Grupo II) y talla (Grupo II) se observó mayor frecuencia de infección. Mediante el análisis de dispersión bivariado se determinó que existe asociación entre peso y prevalencia así como entre longitud y prevalencia parasitaria por Hymenolepis diminuta.

Respecto a la localización del parásito en el hospedador, en 21 ratas, los parásitos se encontraban en el intestino delgado; en tanto que sólo en un roedor los 6 céstodos estuvieron localizados en el intestino grueso (Tabla 1).

La intensidad de la infección parasitaria varió entre 1 y 24 ejemplares por hospedero (Tabla 2). Los individuos adultos de $H$. diminuta hallados en las ratas capturadas y que presentaban proglótidos grávidos, midieron entre 51 y $72 \mathrm{~cm}$ de largo por $0,2 \mathrm{~cm}$ de ancho.

El 85,2\% de ratas estuvo infestada por Xenopsylla cheopis. La intensidad de infestación varió entre 1 y 57 . De las 158 pulgas

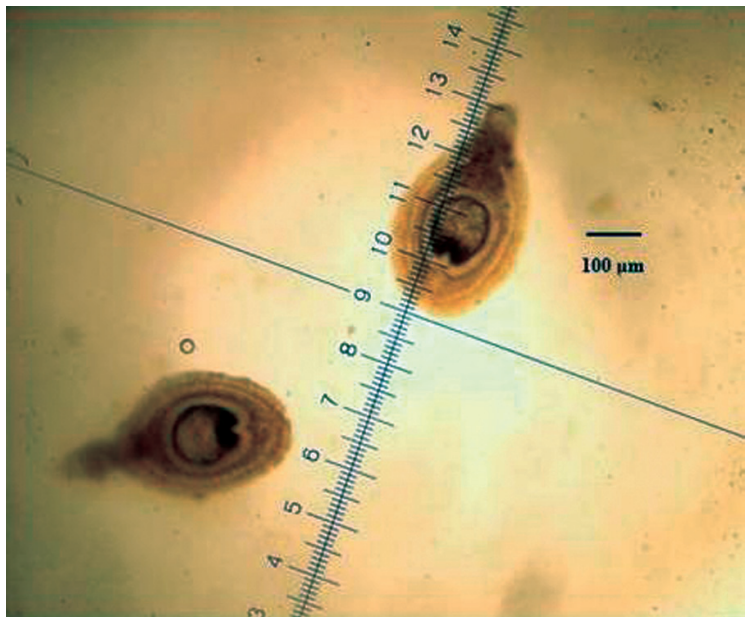

Figura 1. Cisticercoide de Hymenolepis diminuta, encontrado en hemocele de Xenopsylla cheopis, Lima, Perú. 
Tabla 3. Cisticercoides de Hymenolepis diminuta en hemocele de Xenopsylla cheopis, Lima-Perú.

\begin{tabular}{|c|c|c|c|c|c|}
\hline \multirow[b]{2}{*}{ Parásitos } & \multirow{2}{*}{$\begin{array}{c}\text { Prevalencia en } \\
\text { pulgas }(\%) n=158\end{array}$} & \multirow{2}{*}{ Frecuencia total } & \multicolumn{2}{|c|}{ Intensidad } & \multirow{2}{*}{ Localización } \\
\hline & & & Mayor & Menor & \\
\hline Larva cisticercoide de H.diminuta & 12 & 19 de 158 pulgas & 2 & 1 & Hemocele \\
\hline
\end{tabular}

recogidas, el $12 \%$ albergaba cisticercoides en su hemocele (Fig. 1, Tabla 3).

También se observó que a partir de los 18 días, las ratas albinas, a las que se inoculó cisticercoides, comenzaron a eliminar los característicos huevos de Hymenolepis diminuta. Las ratas infectadas experimentalmente, al ser sacrificadas a los 30 días, presentaron en su intestino individuos adultos del céstodo Hymenolepis diminuta.

\section{Discusión}

La presencia de Hymenolepis diminuta en Rattus norvegicus ha sido reportada en diversos estudios desarrollados en Lima. Un informe del año 1947 (Ayulo \& Dammert 1948) señalaba que el $12,7 \%$ de ratas de Lima estaban parasitadas por este céstodo. En el 2002, Iannacone y Alvarińo encontraron $H$. diminuta en $84 \%$ de ratas examinadas del distrito de San Juan de Lurigancho en Lima, número similar al encontrado en el presente estudio $(81,5 \%)$.

En concordancia con Mafiana et al. (1997) quien observó que la talla de $R$. rattus influyó en la prevalencia de infección de $H$. diminuta, en El Cercado de Lima se observó que la mayor longitud y peso de las ratas estuvo relacionada con mayor prevalencia del céstodo.

Khanum et al. (2009) observaron adultos de $H$. diminuta en el ciego de 20,83\% (10 de 48) de ratas infectadas naturalmente (Rattus norvegicus, cepa Long-Evans). En el presente estudio, 21 roedores albergaban a los céstodos en el intestino delgado, en tanto que en una rata sus seis céstodos estuvieron localizados en el intestino grueso. Este último hallazgo, semejante al reportado por Khanum et al., indica que los escólices de estos hymenolepídidos, aunque en menor proporción, pueden alojarse en esta región del tubo digestivo y desarrollar su estróbila.

En lo referente a la intensidad de la infección parasitaria, un alto porcentaje de roedores $(36,4 \%)$ albergaba un solo individuo de $H$. diminuta en su tubo intestinal; en textos de Parasitología se señala que es frecuente que algunos céstodos se encuentren solos en el intestino y ocurra fertilización cruzada entre sus proglótidos.

En numerosas publicaciones científicas y en manuales de enseñanza de Parasitología se asume que los coleópteros de los géneros Tenebrio y Tribolium son los más importantes hospederos intermediarios del céstodo Hymenolepis diminuta, no considerándose la importancia de Xenopsylla cheopis en su ciclo.

Existen pocos estudios sobre el papel de la pulga de la rata en la hymenolepiosis por H.diminuta, así Yamada et al. (1936) y Cáceres y Guillén (1972) lograron infectar larvas de Xenopsylla cheopis con huevos de esa tenia; sin embargo ambos grupos de investigadores no encontraron pulgas naturalmente infectadas. Los resultados del presente trabajo son concluyentes y constituyen el primer registro del rol de $X$. cheopis como hospedero intermediario natural en nuestro país, por lo que es necesario reconsiderar los factores de riesgo y las medidas de prevención en Lima, donde las ratas infectadas con adultos de $H$. diminuta y cuyas pulgas parasitadas con el estadio larvario cisticercoide

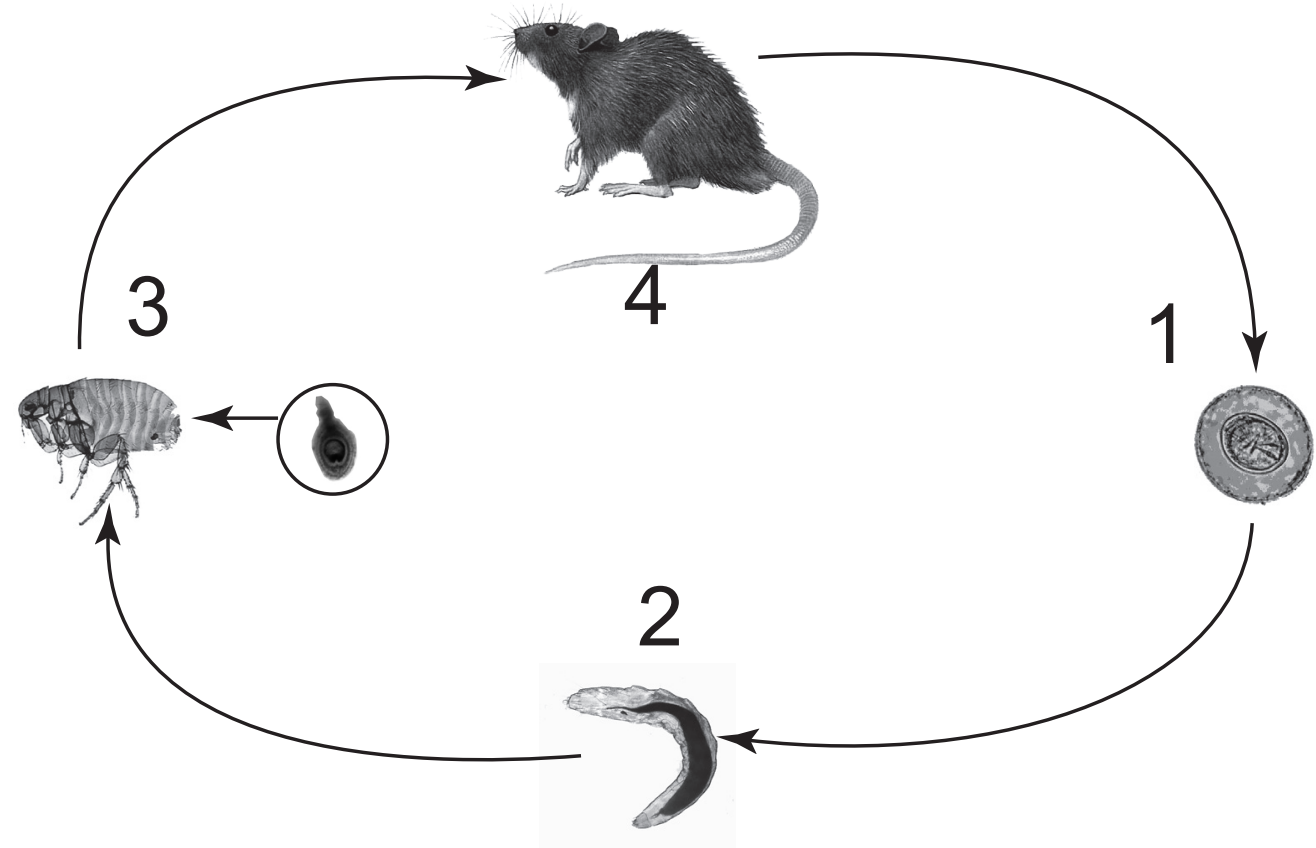

Figura. 2 Ciclo biológico de Hymenolepis diminuta en Lima, Perú. (1) Huevo embrionado, (2) Larva de Xenopsylla cheopis, (3) Hospedero intermediario: $X$. cheopis con cisticercoide en hemocele, (4) Hospedero definitivo: Rattus novergicus. 
constituyen un grave riesgo para la población humana. Respecto al ciclo biológico de Hymenolepis diminuta en Perú, se presenta el esquema desarrollado en la Figura 2, fundamentado en los hallazgos de la presente investigación.

\section{Literatura citada}

Arrojo L., M. Tantaleán. \& J. Huanca-M. 2004. Registro de nuevo huésped intermediario de Hymenolepis diminuta (Cestoda) en el Perú. Rev. peru. biol. 11(1): 107 - 108.

Ayulo R. Víctor M. \& Olga Dammert . 1947. Survey del parasitismo intestinal de las ratas grises (Mus norvegicus) en la ciudad de Lima. Revista Peruana de Medicina Experimental y Salud Pública. 6 (1-4): 76-93.

Cáceres I. \& Z. Guillén de Tantaleán. 1972. Insectos de Lima relacionados con el cisticercoide de Hymenolepis diminuta (Rudolphi, 1819), (Cestoda: Hymenolepididae). Rev. per. entom. 15(1): 142-147.

DIGESA (Dirección General de Salud Ambiental Ministerio de Salud). 2002. Manual de campo para la vigilancia entomológica, $142 \mathrm{pp}$.

Huiza A., M. Tantaleán \& D. Juárez. 1986. Observaciones sobre la presencia de enteroparásitos en la región de Rupa Rupa, departamento de Huanuco. Boletín de Medicina Tropical (Lima) 5:7-9.

Iannacone J. \& L. Alvariño 2002. Helmintofauna de Rattus rattus (Linnaeus, 1978) y Rattus norvegicus (Berkenhout, 1769 (Rodentia: Muridae) en el distrito de San Juan De Lurigancho, Lima- Perú. Revista Peruana de Medicina Experimental y Salud Pública. 19:136-141.
Khanum H., F. Muznebin \& N. Zaibun. 2009. Nematode and Cestode Prevalence, Organal Distribution and Histological Effects Due to Parasitic Infection in Laboratory Rat Strain, LongEvans (Rattus norvegicus Berkenhout, 1769). Bangladesh J. Sci. Ind. Res. 44(2):207-210.

Joyeux, Ch., 1916.-Sur le cycle evolutif de quelques Cestodes. Bull. Soc. Path. Exot. 9:578-583

Mafiana CF, MB Osho \& S. Sam-Wobo. 1997. Gastrointestinal helminth parasites of the black rat (Rattus rattus) in Abeokuta, southwest Nigeria. J Helminthol. 71: 217-20.

Náquira, C., E. Delgado, M. Tantaleán, F. Náquira \& A. Elliot. 1973. Prevalencia de enteroparásitos en escolares de los distritos de San Juan y Magdalena (Departamento de Cajamarca) 1971. Rev. per. med. trop. U.N.M.S.M. 2: 37-41.

Pozo, E. J, G. Troncos C., A. Palacios et al. 2005. Distribución y Hospederos de pulgas (Siphonaptera) en la Provincia de Ayabaca, Piura - 1999. Revista Peruana de Medicina Experimental y Salud Pública. 22(4):316-320

Roberts L., J. Janovy, G. Schmidt \& S. Larry 2009. Roberts'Foundations of Parasitology $8^{\text {th }}$ ed. McGraw-Hill. $701 \mathrm{pp}$.

Smyth J.D. 1963. Biological of cestode of the life-cycle. Technical Communication Number 34, Commonwealth Agricultural Bureau St. Alban England No 34. 38pp.

Traub R. 1952. Record and descriptions of fleas from Perú (Siphonaptera). Proc Entomol; 36(3): 270-3.

Yamada, S., J. Asada \& I. Miyada. 1936. Studies on the life history of a common rat tapeworm, Hymenolepis diminuta (Rudolphi), especially on the relation between this tapeworm and rat fleas. Zool. Mag. (Japan) 48: 437-457. 